\title{
An extremely rare complication after appendectomy in a child: indicators for omental abscess, CRP and Leukocytosis
}

\author{
Sevgi Buyukbese Sarsu ${ }^{1 *}$,
}

\begin{abstract}
A 14 year-old male child underwent appendectomy, and partial omentectomy with the indication of perforated appendicitis. Despite antibiotic therapy at postoperative sixth day, purulent discharge started to ooze from the incision site. Upon detection of a palpable mass in the lower right abdominal quadrant, ultrasonographic examination was performed on the postoperative 14th day which revealed a non-homogeneous hypoechoic mass measuring 50-45 mm with irregular contours. The case was re-operated and a greater omental abscess was found which resected en-bloc. Any evidence of foreign object or residual appendiceal tissue was not found. Postoperative course was uneventful, and the patient was discharged on the 36th postoperative day. Herein, we reported an extremely rare case of omental abscess developed following appendectomy.

Keywords: Appendicitis; Appendectomy; Complications; Omental abscess; Child
\end{abstract}

\section{Introduction}

Development of intraabdominal abscess following appendectomy is an important and potentially fatal complication which requires surgical intervention. Pelvic, subdiaphragmatic, interintestinal, subhepatic, retrocecal, intramesenteric abscesses can develop. Greater omental abscess is an extremely rare condition among intraabdominal abscesses. As reported in previous publications, it can develop on postoperative 16. day (1) or 2 years after the appendectomy (2). Omental abscess is rarely considered in the differential diagnosis of appendectomized patients. This case was reported so as to remind this possibility

\section{Case}

A 14-year-old male adolescent was admitted to the Department of Pediatric Surgery of Cengiz Gökcek Obstetrics and Children's Hospital with typical symptoms of appendicitis on November 9, 2015, 96 hours after onset of his complaints . Laboratory parametres were within normal limits, excepting leucocytosis (19.8 (n:4.5-11) 103/ $\mu \mathrm{L}$, and increased CRP 21.6 (n: < 0.6) mg/dl level. Laparatomy for appendectomy was performed through modified Rockey Davis incision. As soon as the peritoneum was opened, abundant purulent discharge was drained from the right iliac fossa, and pelvis. Appendix vermiformis was wrapped with greater omentum. A calcified appendicolith dropped into pelvis from gangrenous, and perforated appendix which was extracted immediately.
Since omentum was hyperemic, and fibrinous, partial omental resection was performed following typical appendectomy. The inflamed area of the omentum was excised and ligated using 3/0 vicryl sutures. A drain was placed in the pelvic cavity, and surgical wound was closed with sutures. Antibacterial treatment was administered (twice daily IV injections of $1 \mathrm{~g}$ ceftazidime, and $500 \mathrm{mg}$ metronidazole). The first 5 days body temperature fluctuated between $37.8^{\circ} \mathrm{C}$, and $38.2^{\circ} \mathrm{C}$ from the postoperative 6 th day on purulent discharged oozed from the incision site. Despite initiation of antibiotic therapy (trimethoprim/sulfametoxazol and gentamycin) based on antibiotic susceptibility tests, his health state did not improve, and on the following day, (postoperative 14. day) a palpable mass was detected on the right lower abdominal quadrant. An ultrasound (US) examination revealed a non-homo $\neg$ geneous hypoechoic mass in the right iliac fossa with irregular contours, and measuring $50 \times 45 \mathrm{~mm}$. According to US findings, and typical clinical data, reoperation was planned. Abdominal cavity was opened through the previous incision line, but any purulent collection was not found. Appendectomy loge, and cecum were intact. In the right lower abdominal quadrant greater omentum was palpated as a solid mass Omental mass was excised, and drained purulent material was sent for culture. Greater omentum with dimensions of 4 x 5 $\mathrm{cm}$ was peeled away from the terminal ileum without causing any harm (Fig. 1).

Received 31-01-2016 Accepted 15-02-2016 Available Online 15-05-2016

1 Cengiz Gokcek Obstetrics and Children's Hospital, Dept. of Pediatric Surgery, Gaziantep, Turkey

* Corresponding Author: Sevgi Buyukbese Sarsu E-mail: sarsusevgi@yahoo.com.tr Phone: +90 3423600888 


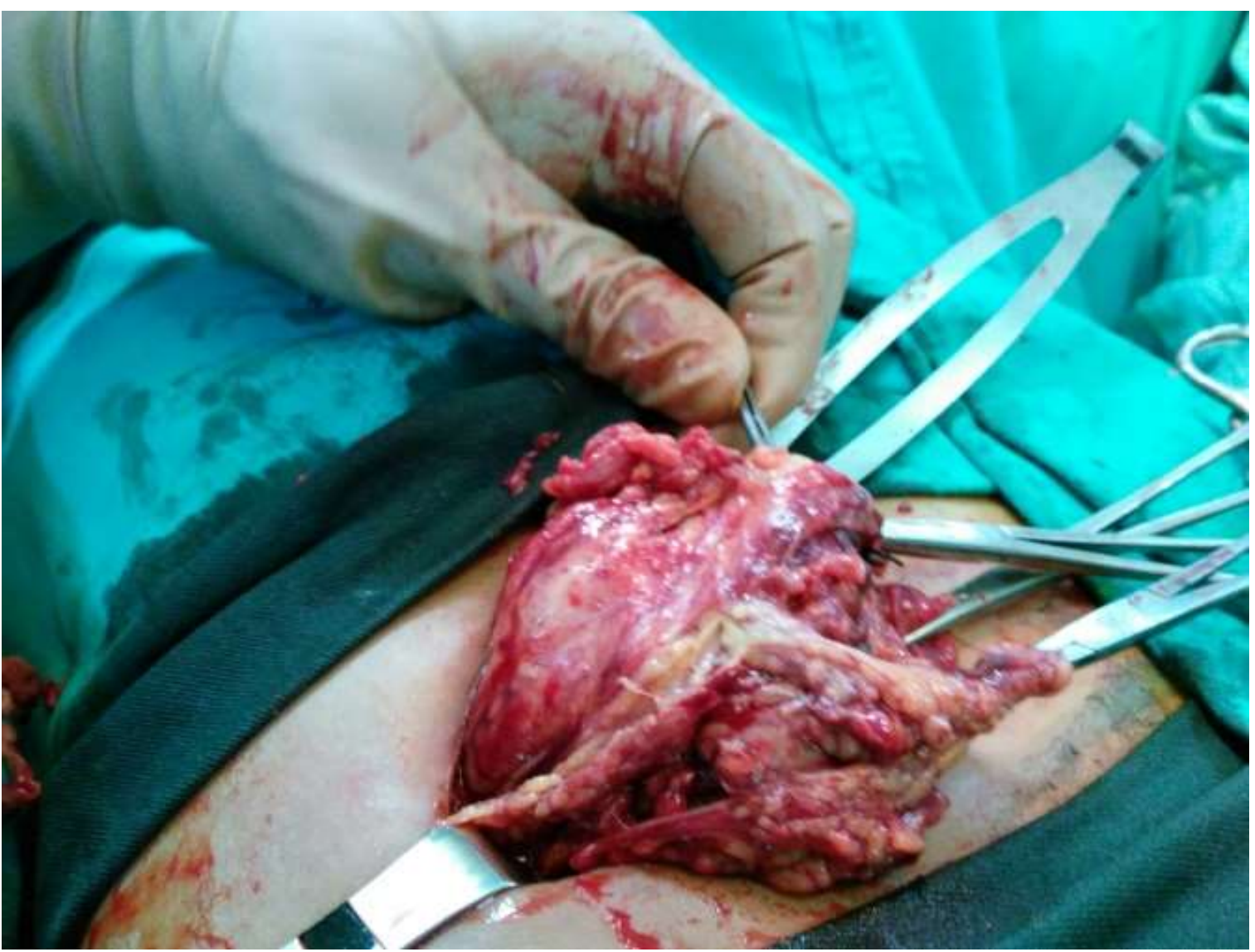

Figure 1: Resected specimen including a part of the greater omentum and the abscess.

Following resection, minimal bleeding was controlled. Based on antibiotic susceptibility test results Escherichia coli sensitive antibiotherapy was initiated. The patient was discharged in a satisfactory condition on the 36 th postoperative day.

\section{Discussion}

Development of abscess following appendectomy is an extremely rare condition which is nowadays thought to be an unacceptable entity by many surgeons. However articles were published on this issue during 1970s, and 1980s (3,4). Omental abscess is a special version of omentitis, and exists in two types. Primary omentitis develops in patients without history of surgery as a complication of omental torsion, ischemia, thromboembolic lesions, while secondary omentitis evolves as a complication of an inflammatory process in another abdominal organ (4). Omentitis can be classified according to the degree of inflammation. Secondary omentitis is more prevalent than primary omentitis, and solitary forms are more widespread than those associated with abscess. Postappendectomy purulent omentitis is an extremely rare entity.
The incidence rates of omental abscess have been reported as $0.011 \%$ by Cortesi et al, (5), and as $0.02 \%$ by Bairov and Golovanov (6). Paliuga reported development of intraabdominal abscesses in three different locations including greater omentum in one of their patients (7).

Almost all patients who developed purulent omentitis during postoperative period of appendectomy have been reported as cases with complicated appendicitis, and destructive (usually gangrenous) appendicitis associated with peritonitis $(4,6,8)$. Most of the cases consisted of patients who had undergone primary appendectomy combined with omentectomy performed because of secondary inflammatory involvement of greater omentum (8). Clinical symptoms of omental abscess include abdominal pain, fever and systemic inflammation. On palpation local tenderness, and most of the time a mass is palpated. This condition does not develop immediately after appendectomy; it can reportedly emerge between 16 days, and 2 years after appendectomy. $(1,2)$. Omental abscess developed long after appendectomy may be mistaken as an intraabdominal tumor (2). Indolent omental inflammation may be provoked in some cases. (1). 
For diagnosis, as previously reported in many publications US, CT, and MRI may be used widely in clinical practice. Although some authors have thought that US has a lower diagnostic value in omental abscesses localized close to the abdominal wall (8), we consider US as a valuable diagnostic tool. Electromyography had been used once in the diagnosis of secondary omentitis, however nowadays it an outmoded diagnostic modality. In our case we made the diagnosis of omentitis using only US. In challenging cases, CT may also use locally. (2). Other omental lesions include omental torsion, and infarct (9).

The moist effective treatment of omental abscess is its removal together with healthy tissue around the welldefined contours of the mass lesion. Drainage of the abscess cavity is another surgical treatment method which should be reserved for special indications which may jeopardize intestinal loops. Laparoscopic drainage may be less invasive in cases with primary omental abscess (10). In our case ileal segments in the omental mass forced us to prefer open surgery rather than laparoscopic approaches in order to prevent intestinal damage. US-guided drainage was not considered due to risk of recurrence of abscess. Since omental abscess developed secondary to infected ligature, drainage of abscess without resection wouldn't be an appropriate approach for our patient. In cases that developed abscess secondary to fecaliths, radical treatment involves opening of abscess, and extraction of the fecalith. Otherwise, possibility of recurrence is very high. (11). Up to now optimal treatment of omental abscess still continues to be omental resection $(2,12)$.

Prevention of purulent omentitis is possible with correct application of omental resection. Omentum should be resected in small parts, and leaving large remnants behind should be avoided (4). The resection line should be extended so as to include non-inflamed, intact tissues $(4,6)$.

Herein, an extremely rare condition with development of greater omental abscess during postoperative period of a patient who underwent appendectomy with the indication of perforated appendicitis was reported. Among intraabdominal abscesses, the possibility of greater omental abscess which has faded into oblivion recently should be always kept in mind as a postoperative complication.

Conflict of Interest: The authors declare no potential conflicts of interest with respect to the research, authorship, and/or publication of this article.
Ethical issues: All Authors declare that Originality of research/article etc... and ethical approval of Research/Case and responsibilities of research against local ethics commission are under the Authors responsibility. The study was completed due to defined rules by the Local Ethics Commission guidelines and audits.

\section{References}

1. Shiryajev NY, Glebova VA, Bernstein MA, Kokhaneo NY. A Very Rare Complication After Appendectomy in a Young Adult Patient: Abscess of the Greater Omentum J Med Cases. 2014;5(1):36-9

2. Hung CC, Chou CM, Chen HC. An omental abscess mimicking an intra-abdominal tumor. J Chin Med Assoc. 2012;75(3):136-8.

3. Chukhrienko DP, Bereznitskii IS. Intraabdominal Ab-scesses and Phlegmons. Kyiv, USSR: Zdorov'ya, 1977:1136 (in Russian).

4. Odinak VM, Gridina GI. (Omentitis in children). Kh-irurgiia (Mosk). 1987;(8):54-8.

5. Cortesi N, Manenti A, Rossi A, Zanni C, Barberini G, Gibertini G. L'appendicite aigue et ses complications postoperatoires. A propos d'une serie de 8738 cas. J Chir (Paris). 1985;122(10):577-9

6. Bairov GA, Golovanov la S. (Infiltrative subacute omentitis in children with acute appendicitis). Vestn Khir Im I I Grek. 1988;140(1):116-20.

7. Paliuga NI. (Abscess of the greater omentum after ap-pendectomy). Vestn Khir Im I I Grek. 1983;130(4):6970.

8. Derzhavin VM, Ivanova MN, Gordeeva IP, Konovalov AK. Secondary acute omentites in children. Vopr Okhr Materin Det. 1986;31(8):29-32 (in Russian).

9. Joshi S, Cuthbert GA, Kerwat R Omental torsion, a rare cause of acute abdomen. BMJ Case Rep. 2016: 5;2016

10. De Brabandere K, Vanpaemel G, Verheyen L. Sponta $\neg$ neous abscesses of the abdominal wall, omentum and abdominal cavity caused by group $\mathrm{G}$ streptococci: a case report. Acta Chir Belg. 2008;108(6):765-7.

11. Buckley O, Geoghegan T, Ridgeway P, Colhoun E, Snow A, Torreggiani WC. The usefulness of CT guided drainage of abscesses caused by retained appendicoliths. Eur J Radiol. 2006;60(1):80-3.

12. Lawson GA, 3rd, Castaldo ET, Miller RS. Primary omental abscess caused by Streptococcus constellatus: a case report. Surg Infect (Larchmt). 2010;11(3):339-41. 